\title{
Forced Vital Capacity in 6 Seconds
}

National Cancer Institute

\section{Source}

National Cancer Institute. Forced Vital Capacity in 6 Seconds. NCI Thesaurus. Code C124421.

The volume of gas a subject can forcefully exhale during the first six seconds following maximum inhalation. 